\title{
Existence and stability of solitary waves for the generalized Korteweg-de Vries equations
}

Mingli Hong*

\section{"Correspondence:}

hml001@sohu.com

Institute of Disaster Prevention,

Sanhe Hebei, 065201, China

\begin{abstract}
In this paper, we consider the fractional Korteweg-de Vries equations with general nonlinearities. By studying constrained minimization problems and applying the method of concentration-compactness, we obtain the existence of solitary waves for the generalized Korteweg-de Vries equations under some assumptions of the nonlinear term. Moreover, we prove that the set of minimizers is a stable set for the initial value problem of the equations, in the sense that a solution which starts near the set will remain near it for all time.
\end{abstract}

Keywords: generalized Korteweg-de Vries equations; constrained minimization problems; concentration-compactness; stability

\section{Introduction}

This paper is devoted to studying the existence and stability of solitary wave solutions of the generalized Korteweg-de Vries equation

$$
u_{t}+(f(u))_{x}-(L(u))_{x}=0 \quad \text { in } \mathbb{R}
$$

where $f(u)$ satisfies the following assumption:

(A) $f(u) \in \mathcal{C}(\mathbb{R}, \mathbb{R}), \lim _{u \rightarrow 0} \frac{f(u)}{|u|}=0$ and $\lim _{|u| \rightarrow \infty} \frac{f(u)}{|u|^{\gamma}}=0$ for some $1<\gamma<1+4 \alpha$,

$$
\widehat{L(u)}(\xi)=|\xi|^{2 \alpha} \widehat{u}(\xi)
$$

$0<\alpha \leq 1$, the Fourier transform $\mathcal{F} \psi(\xi)=\widehat{\psi}(\xi)=\frac{1}{(2 \pi)^{\frac{1}{2}}} \int_{\mathbb{R}} u(x) e^{-i \xi \cdot x} d x$.

When $f(u)=\frac{1}{2} u^{2}$ and $\alpha=1$, equation (1.1) is the well-known Korteweg-de Vries equation, introduced by Korteweg and de Vries in 1895 (cf. [1]). The existence and stability of solitary waves of the Korteweg-de Vries equation is considered by Benjamin in [2]. Recently, in [3], Pelinovsky obtained a Korteweg-de Vries equation with a forcing term, which is a simple analytical model of tsunami generation by submarine landslides.

Here, we consider the generalized Korteweg-de Vries equation (1.1). Let $F(u)=\int_{0}^{u} f(s) d s$. Since the functionals

$$
E(u)=\int_{-\infty}^{+\infty}\left[\frac{1}{2} u L(u)-F(u)\right] d x
$$

(0) 2013 Hong; licensee Springer. This is an Open Access article distributed under the terms of the Creative Commons Attribution License (http://creativecommons.org/licenses/by/2.0), which permits unrestricted use, distribution, and reproduction in any medium, provided the original work is properly cited. 
and

$$
Q(u)=\frac{1}{2} \int_{-\infty}^{+\infty} u^{2} d x
$$

are two conserved quantities with (1.1), for studying the existence of solitary wave solutions to (1.1), by the variational methods, the solitary wave solutions to equation (1.1) will be founded as minimizers of

$$
I_{q}:=\inf \left\{E(u) ; u \in H^{1}(\mathbb{R}), Q(u)=q\right\},
$$

where $q>0$. Denote the set of minimizers of the problem $\left(I_{q}\right)$ by

$$
G_{q}:=\left\{u ; u \in H^{1}(\mathbb{R}), E(u)=I_{q}, Q(u)=q\right\} .
$$

Inspired by the methods used in $[4,5]$, by studying the problem $\left(I_{q}\right)$, we obtain the existence of solitary waves for equation (1.1) with some special nonlinearities $f(u)=\frac{1}{p} u^{p}$, where $1<p<1+4 \alpha$, and general nonlinearities satisfying the assumption (A). Moreover, we prove that the set $G_{q}$ of minimizers is a stable set for the initial value problem of equation (1.1) in the sense that a solution which starts near the set will remain near it for all time. In order to obtain those results, we have to overcome one main difficulty: the minimization problem $\left(I_{q}\right)$ is given in the unbounded domain $\mathbb{R}$ which results in the loss of compactness. As is done in [4, 6], we overcome the difficulty of loss of compactness by the method of concentration-compactness introduced by Lions in $[7,8]$ for solving some minimization problems in unbounded domains.

Now we give our main results.

Theorem 1.1 Suppose that $\alpha=1$ and $f(u)$ satisfies condition (A) and $I_{q_{0}}<0$ for some $q_{0}>0$. Then there exists $0<q^{*} \leq q_{0}$ such that $G_{q^{*}}$ is not empty. Moreover, if $\left\{u_{n}\right\}$ is a minimizing sequence for the problem $\left(I_{q^{*}}\right)$, then there exist a sequence $\left\{y_{n}\right\} \subset \mathbb{R}$ and $g \in G_{q^{*}}$ such that $\left\{u_{n}\left(\cdot+y_{n}\right)\right\}$ contains a subsequence converging strongly in $H^{1}(\mathbb{R})$ to $g$, and

$$
\lim _{n \rightarrow+\infty} \inf _{g \in G_{q^{*}}}\left\|u_{n}-g\right\|=0
$$

where $\|\cdot\|$ is the norm of $H^{1}(\mathbb{R})$.

Theorem 1.2 Under the assumptions of Theorem 1.1, the set $G_{q^{*}}$ is $H^{1}(\mathbb{R})$-stable with respect to equation (1.1), i.e., for any $\varepsilon>0$, there exists $\delta>0$ such that if

$$
\inf _{g \in G_{q^{*}}}\left\|u_{0}-g\right\|<\delta
$$

then the solution $u(x, t)$ to equation (1.1) with initial data $u_{0}$ satisfies

$$
\inf _{g \in G_{q^{*}}}\|u(t, \cdot)-g\|<\varepsilon
$$

for any $t \in[0, T)$. 
Theorem 1.3 Suppose that $0<\alpha<1$ and $f(u)$ satisfies condition (A) and $I_{q_{0}}<0$ for some $q_{0}>0$. Then there exists $0<q^{*} \leq q_{0}$ such that $G_{q^{*}}$ is not empty. Moreover, if $\left\{u_{n}\right\}$ is a minimizing sequence for the problem $\left(I_{q^{*}}\right)$, then there exists a sequence $\left\{y_{n}\right\} \subset \mathbb{R}$ and $g \in G_{q^{*}}$ such that $\left\{u_{n}\left(\cdot+y_{n}\right)\right\}$ contains a subsequence converging strongly in $H^{\alpha}(\mathbb{R})$ to $g$, and

$$
\lim _{n \rightarrow+\infty} \inf _{g \in G_{q^{*}}}\left\|u_{n}-g\right\|_{\alpha, 2}=0
$$

where $\|\cdot\|_{\alpha, 2}$ is the norm of $H^{\alpha}(\mathbb{R})$ given in Section 5.

Theorem 1.4 Under the assumptions of Theorem 1.1, the set $G_{q^{*}}$ is $H^{\alpha}(\mathbb{R})$-stable with respect to equation (1.1), i.e., for any $\varepsilon>0$, there exists $\delta>0$ such that if

$$
\inf _{g \in G_{q^{*}}}\left\|u_{0}-g\right\|_{\alpha, 2}<\delta
$$

then the solution $u(x, t)$ to equation (1.1) with initial data $u_{0}$ satisfies

$$
\inf _{g \in G_{q^{*}}}\|u(t, \cdot)-g\|_{\alpha, 2}<\varepsilon
$$

for any $t \in[0, T)$.

The paper is organized as follows. In Section 2, we give some preliminaries. In Section 3, we study the existence and stability of solitary waves of equation (1.1) with some special nonlinearities $f(u)=\frac{1}{p} u^{p}$. Section 4 is devoted to studying equation (1.1) with general nonlinearities $f(u)$ satisfying the assumption (A). We shall consider the existence and stability of solitary waves of equation (1.1) with $0<\alpha<1$ in Section 5 .

\section{Some preliminaries}

At first, we give some notations. The set of all integers and the set of all real numbers are written as $\mathbb{Z}$ and $\mathbb{R}$, respectively. And all the integrals will be taken over $\mathbb{R}$ unless specified. $L^{p}(\mathbb{R})$ denotes the usual Lebesgue space with the norm $|\cdot|_{p}$ given by

$$
|\cdot|_{p}=\left(\int|u|^{p} d x\right)^{\frac{1}{p}} \text { for } 1 \leq p<+\infty .
$$

The Sobolev space $H^{1}(\mathbb{R})$ is defined by

$$
H^{1}(\mathbb{R}):=\left\{u: u \in L^{2}(\mathbb{R}) \text { and } u_{x} \in L^{2}(\mathbb{R})\right\}
$$

whose norm is given by

$$
\|\cdot\|=\left(\int\left(\left|u_{x}\right|^{2}+|u|^{2}\right) d x\right)^{\frac{1}{2}} .
$$

Now, we give Lemma 2.1 and Lemma 2.2 which will be used to study the behavior of the minimizing sequence for the problem $\left(I_{q}\right)$. Lemma 2.2 is due to Lions $[7,8]$. 
Lemma 2.1 Suppose that $B>0$ and $\delta>0$ are given. Then there exists $\eta=\eta(B, \delta)$ such that if $u \in H^{1}(\mathbb{R})$ with $\|u\| \leq B$ and $|u|_{p+1} \geq \delta$, then

$$
\sup _{y \in \mathbb{R}} \int_{y-\frac{1}{2}}^{y+\frac{1}{2}}|u|^{p+1} d x \geq \eta
$$

Proof We have

$$
\sum_{j \in \mathbb{Z}} \int_{j-\frac{1}{2}}^{j+\frac{1}{2}}\left[\left(u_{x}\right)^{2}+u^{2}\right] d x=\|u\|^{2} \leq \frac{B^{2}}{|u|_{p+1}^{p+1}}|u|_{p+1}^{p+1}=\sum_{j \in \mathbb{Z}} \frac{B^{2}}{|u|_{p+1}^{p+1}} \int_{j-\frac{1}{2}}^{j+\frac{1}{2}}|u|^{p+1} d x .
$$

Therefore, there exists some $j_{0} \in \mathbb{Z}$ such that

$$
\int_{j_{0}-\frac{1}{2}}^{j_{0}+\frac{1}{2}}\left[\left(u_{x}\right)^{2}+u^{2}\right] d x \leq \frac{B^{2}}{|u|_{p+1}^{p+1}} \int_{j_{0}-\frac{1}{2}}^{j_{0}+\frac{1}{2}}|u|^{p+1} d x .
$$

Applying the Sobolev embedding theorem [9], there exists a constant $A$ such that

$$
\begin{aligned}
\left(\int_{j_{0}-\frac{1}{2}}^{j_{0}+\frac{1}{2}}\left[\left(u_{x}\right)^{2}+u^{2}\right] d x\right)^{\frac{1}{p+1}} & \leq A\left(\int_{j_{0}-\frac{1}{2}}^{j_{0}+\frac{1}{2}}\left[\left(u_{x}\right)^{2}+u^{2}\right] d x\right)^{\frac{1}{2}} \\
& \leq \frac{A B}{|u|_{p+1}^{\frac{p+1}{2}}}\left(\int_{j_{0}-\frac{1}{2}}^{j_{0}+\frac{1}{2}}|u|^{p+1} d x\right)^{\frac{1}{2}} .
\end{aligned}
$$

Thus, we obtain

$$
\int_{j_{0}-\frac{1}{2}}^{j_{0}+\frac{1}{2}}|u|^{p+1} d x \geq\left(\frac{|u|_{p+1}^{\frac{p+1}{2}}}{A B}\right)^{\frac{2(p+1)}{p-1}} \geq \frac{\delta^{\frac{(p+1)^{2}}{p-1}}}{(A B)^{\frac{2(p+1)}{p-1}}}
$$

Taking $\eta=\frac{\delta \frac{(p+1)^{2}}{p-1}}{(A B)^{\frac{2(p+1)}{p-1}}}$, it follows that

$$
\sup _{y \in \mathbb{R}} \int_{y-\frac{1}{2}}^{y+\frac{1}{2}}|u|^{p+1} d x \geq \int_{j_{0}-\frac{1}{2}}^{j_{0}+\frac{1}{2}}|u|^{p+1} d x \geq \eta
$$

Lemma 2.2 Let $\left\{u_{n}\right\}$ be a bounded sequence in $H^{1}(\mathbb{R})$ such that

$$
\sup _{y \in \mathbb{R}} \int_{y-1}^{y+1}\left|u_{n}\right|^{2} d x \rightarrow 0 \quad \text { as } n \rightarrow+\infty
$$

for some $r>0$. Then $u_{n} \rightarrow 0$ in $L^{s}(\mathbb{R})$ for $2<s<\infty$.

Proof Let $2<s<\infty$. Without loss of generality, we may assume $r=1$. It follows from the interpolation inequalities that

$$
|u|_{L^{s}(B(y, 1))} \leq A|u|_{L^{2}(B(y, 1))}^{\frac{s+2}{2}}\|u\|_{H^{1}(B(y, 1))}^{\frac{s-2}{2}}
$$

where $A>0$ is a constant independent of $u$. 
Covering $\mathbb{R}$ by a family of intervals $\left(y_{i}-1, y_{i}+1\right)$ such that each point of $\mathbb{R}$ is contained in at most two such intervals and summing this inequality over this family of intervals, we get

$$
|u|_{L^{s}}^{s} \leq 2 A\left(\sup _{y \in \mathbb{R}} \int_{y-1}^{y+1}|u|^{2} d x\right)^{\frac{s+2}{4}}\|u\|^{\frac{s-2}{2}} .
$$

Since $\left\{u_{n}\right\}$ is bounded in $H^{1}(\mathbb{R})$ and $\sup _{y \in \mathbb{R}} \int_{y-1}^{y+1}\left|u_{n}\right|^{2} d x \rightarrow 0$ as $n \rightarrow+\infty$, applying the above inequality, we know that $u_{n} \rightarrow 0$ in $L^{s}(\mathbb{R})$ for $2<s<\infty$.

Next, we establish a convergence result that will be used in the proof of Theorem 1.3.

Lemma 2.3 Let $f \in C(\mathbb{R}, \mathbb{R})$ and suppose that

$$
|f(t)| \leq C\left(|t|+|t|^{p_{1}}\right) \quad \text { for all } t \in \mathbb{R},
$$

where $1<p_{1}<\infty$. If $u_{n} \rightarrow u_{0}$ in $H^{1}(\mathbb{R})$ and $u_{n} \rightarrow u_{0}$ a.e. on $\mathbb{R}$, then

$$
\lim _{n \rightarrow \infty}\left[\int_{-\infty}^{+\infty} F\left(u_{n}\right) d x-\int_{-\infty}^{+\infty} F\left(u_{0}\right) d x-\int_{-\infty}^{+\infty} F\left(u_{n}-u_{0}\right) d x\right]=0,
$$

where $F(u)=\int_{0}^{u} f(s) d s$.

Proof Let $R>0$. Applying the mean value theorem, we have

$$
\begin{aligned}
& \int_{-\infty}^{+\infty} F\left(u_{n}\right) d x \\
& \quad=\int_{|x|<R} F\left(u_{n}\right) d x+\int_{|x| \geq R} F\left(u_{0}+\left(u_{n}-u_{0}\right)\right) d x \\
& \quad=\int_{|x|<R} F\left(u_{n}\right) d x+\int_{|x| \geq R}\left[F\left(u_{n}-u_{0}\right) d x+f\left(u_{n}-u_{0}+\theta u_{0}\right) u_{0}\right] d x
\end{aligned}
$$

where $0<\theta<1$ is dependent on $x$ and $R$. Now we write

$$
\begin{aligned}
& \left|\int_{-\infty}^{+\infty} F\left(u_{n}\right) d x-\int_{-\infty}^{+\infty} F\left(u_{0}\right) d x-\int_{-\infty}^{+\infty} F\left(u_{n}-u_{0}\right) d x\right| \\
& \quad \leq\left|\int_{|x|<R}\left[F\left(u_{n}\right)-F\left(u_{0}\right)\right] d x\right|+\left|\int_{|x| \geq R} F\left(u_{0}\right) d x\right|+\left|\int_{|x|<R} F\left(u_{n}-u_{0}\right) d x\right| \\
& \quad+\left|\int_{|x| \geq R} f\left(u_{n}-u_{0}+\theta u_{0}\right) u_{0} d x\right| .
\end{aligned}
$$

It follows from (2.1), the mean value theorem and the Hölder inequality that

$$
\begin{aligned}
& \left|\int_{|x|<R}\left[F\left(u_{n}\right)-F\left(u_{0}\right)\right] d x\right| \\
& \quad=\left|\int_{|x|<R} f\left(u_{0}+\theta\left(u_{n}-u_{0}\right)\right)\left(u_{n}-u_{0}\right) d x\right|
\end{aligned}
$$


Hong Boundary Value Problems 2013, 2013:121

Page 6 of 17

http://www.boundaryvalueproblems.com/content/2013/1/121

$$
\begin{aligned}
\leq & C \int_{|x|<R}\left|u_{0}+\theta\left(u_{n}-u_{0}\right)\right|\left|u_{n}-u_{0}\right| d x \\
& +C \int_{|x|<R}\left|u_{0}+\theta\left(u_{n}-u_{0}\right)\right|^{p_{1}}\left|u_{n}-u_{0}\right| d x \\
\leq & C \int_{|x|<R}\left|u_{0}\right|\left|u_{n}-u_{0}\right| d x+C \int_{|x|<R}\left|u_{n}-u_{0}\right|^{2} d x \\
& +\varepsilon \int_{|x|<R}\left|u_{0}\right|^{p_{1}}\left|u_{n}-u_{0}\right| d x+C_{\varepsilon} \int_{|x|<R}\left|u_{n}-u_{0}\right|^{p_{1}+1} d x \\
\leq & C\left(\int_{|x|<R}\left|u_{0}\right|^{2} d x\right)^{\frac{1}{2}}\left(\int_{|x|<R}\left|u_{n}-u_{0}\right|^{2} d x\right)^{\frac{1}{2}}+C \int_{|x|<R}\left|u_{n}-u_{0}\right|^{2} d x \\
& +\varepsilon\left(\int_{|x|<R}\left|u_{0}\right|^{2 p_{1}} d x\right)^{\frac{1}{2}}\left(\int_{|x|<R}\left|u_{n}-u_{0}\right|^{2} d x\right)^{\frac{1}{2}} \\
& +C_{\varepsilon}\left|u_{|x|<R}-u_{n}\right|^{p_{1}+1} d x
\end{aligned}
$$

and

$$
\left|\int_{|x|<R} F\left(u_{n}-u_{0}\right) d x\right| \leq C \int_{|x|<R}\left|u_{n}-u_{0}\right|^{2} d x+C \int_{|x|<R}\left|u_{n}-u_{0}\right|^{p_{1}+1} d x
$$

Since the embedding $H^{1}(\mathbb{R}) \hookrightarrow L_{\text {lac }}^{s}(\mathbb{R})(2 \leq s<\infty)$ is compact, the inequalities (2.3) and (2.4) imply that

$$
\begin{aligned}
& \left|\int_{|x|<R}\left[F\left(u_{n}\right)-F\left(u_{0}\right)\right] d x\right| \rightarrow 0 \quad \text { as } n \rightarrow \infty, \\
& \left|\int_{|x|<R} F\left(u_{n}-u_{0}\right) d x\right| \rightarrow 0 \quad \text { as } n \rightarrow \infty .
\end{aligned}
$$

Similarly, by the Hölder inequality, the Sobolev embedding theorem and (2.1), we get

$$
\begin{aligned}
& \left|\int_{|x| \geq R} f\left(u_{n}-u_{0}+\theta u_{0}\right) u_{0} d x\right| \\
& \leq C \int_{|x| \geq R}\left|u_{n}-u_{0}+\theta u_{0}\right|\left|u_{0}\right| d x+C \int_{|x| \geq R}\left|u_{n}-u_{0}+\theta u_{0}\right|^{p_{1}}\left|u_{0}\right| d x \\
& \leq C\left(\int_{|x| \geq R}\left|u_{n}\right|^{2} d x\right)^{\frac{1}{2}}\left(\int_{|x| \geq R}\left|u_{0}\right|^{2} d x\right)^{\frac{1}{2}}+C \int_{|x| \geq R}\left|u_{0}\right|^{2} d x \\
& \quad+\varepsilon\left(\int_{|x| \geq R}\left|u_{n}\right|^{2 p_{1}} d x\right)^{\frac{1}{2}}\left(\int_{|x| \geq R}\left|u_{0}\right|^{2} d x\right)^{\frac{1}{2}}+C_{\varepsilon} \int_{|x| \geq R}\left|u_{0}\right|^{p_{1}+1} d x \\
& \quad C|| u_{n}\left\|\left(\int_{|x| \geq R}\left|u_{0}\right|^{2} d x\right)^{\frac{1}{2}}+C \int_{|x| \geq R}\left|u_{0}\right|^{2} d x+\varepsilon\right\| u_{n} \|^{p_{1}}\left(\int_{|x| \geq R}\left|u_{0}\right|^{2} d x\right)^{\frac{1}{2}} \\
& \quad+C_{\varepsilon} \int_{|x| \geq R}\left|u_{0}\right|^{p_{1}+1} d x .
\end{aligned}
$$


Since $\left\{u_{n}\right\}$ is bounded in $H^{1}(\mathbb{R})$, we see that

$$
\left|\int_{|x| \geq R} f\left(u_{n}-u_{0}+\theta u_{0}\right) u_{0} d x\right| \rightarrow 0 \quad \text { as } R \rightarrow \infty
$$

Hence, combining (2.2), (2.5), (2.6) and (2.7), we obtain

$$
\int_{-\infty}^{+\infty} F\left(u_{n}\right) d x-\int_{-\infty}^{+\infty} F\left(u_{0}\right) d x-\int_{-\infty}^{+\infty} F\left(u_{n}-u_{0}\right) d x \rightarrow 0 \quad \text { as } n \rightarrow \infty .
$$

\section{The case of special nonlinearity}

In this section, we only consider the case of $\alpha=1$ and $f(u)=\frac{1}{p} u^{p}$, where $1<p<5$. Correspondingly,

$$
E(u)=\int_{-\infty}^{+\infty}\left[\frac{1}{2}\left(u_{x}\right)^{2}-\frac{1}{p} \frac{1}{p+1} u^{p+1}\right] d x .
$$

At first, we commence by studying some properties of the functional $I_{q}:(0,+\infty) \rightarrow \mathbb{R}$ and the behavior of the minimizing sequences for the problem $\left(I_{q}\right)$.

Lemma 3.1 For any $q>0$,

(i) $-\infty<I_{q}<0$;

(ii) If $\left\{u_{n}\right\}$ is a minimizing sequence for the problem $\left(I_{q}\right)$, there exists a constant $B>0$ such that $\left\|u_{n}\right\| \leq B$ for all $n$;

(iii) If $\left\{u_{n}\right\}$ is a minimizing sequence for the problem $\left(I_{q}\right)$, there exist a positive constant $\delta$ and a sequence $\left\{y_{n}\right\}$ of real numbers such that

$$
\int_{y_{n}-\frac{1}{2}}^{y_{n}+\frac{1}{2}}\left|u_{n}\right|^{p+1} d x \geq \delta
$$

for sufficiently large $n$.

Proof (i) Choose any function $u \in H^{1}(\mathbb{R})$ such that $Q(u)=q$ and $\int u^{p+1} d x \neq 0$. For any $\theta>0$, define $u_{\theta}(x)=\sqrt{\theta} u(\theta x)$. Then we have

$$
Q\left(u_{\theta}\right)=q
$$

and

$$
E\left(u_{\theta}\right)=\frac{1}{2} \theta^{2} \int\left(u_{x}\right)^{2} d x-\frac{1}{p(p+1)} \theta^{\frac{p-1}{2}} \int u^{p+1} d x .
$$

For $1<p<5$, by taking $\theta>0$ sufficiently small, we get $I_{q} \leq E\left(u_{\theta}\right)<0$.

Next we prove $I_{q}>-\infty$. Let $u \in H^{1}(\mathbb{R})$ such that $Q(u)=q$. By the Sobolev embedding theorems and interpolation inequalities, we get

$$
\left|\int u^{p+1} d x\right| \leq|u|_{p+1}^{p+1} \leq A|u|_{\frac{P-1}{2(P+1)}}^{P+1} \leq A\|u\|^{\frac{p-1}{2}}|u|_{2}^{\frac{p+3}{2}},
$$


where $A$ denotes various constants which are independent of $u$. Using the Young inequality, we derive from (3.1)

$$
\left|\int u^{p+1} d x\right| \leq \varepsilon\|u\|^{2}+A_{\varepsilon}|u|_{2}^{\frac{2 p+6}{5-p}} \leq \varepsilon\|u\|^{2}+A_{\varepsilon, q},
$$

where $\varepsilon>0$ is arbitrary and $A_{\varepsilon, q}$ depends on $\varepsilon$ and $q$, but not on $u$. Therefore,

$$
\begin{aligned}
E(u) & =E(u)+Q(u)-Q(u) \\
& =\frac{1}{2}\|u\|^{2}-\frac{1}{p(p+1)} \int u^{p+1} d x-\frac{1}{2} \int u^{2} d x \\
& \geq \frac{1}{2}\|u\|^{2}-\frac{\varepsilon}{p(p+1)}\|u\|^{2}-\frac{1}{p(p+1)} A_{\varepsilon, q}-q .
\end{aligned}
$$

Choosing $\varepsilon<\frac{p(p+1)}{2}$, we obtain the lower bound of the functional $E$

$$
E(u) \geq-\frac{1}{p(p+1)} A_{\varepsilon, q}-q,
$$

which implies $I_{q} \geq-\frac{1}{p(p+1)} A_{\varepsilon, q}-q>-\infty$.

(ii) Let $\left\{u_{n}\right\}$ be a minimizing sequence for the problem $\left(I_{q}\right)$. Then, by (3.1), we have

$$
\begin{aligned}
\frac{1}{2}\left\|u_{n}\right\|^{2} & =E\left(u_{n}\right)+Q\left(u_{n}\right)+\frac{1}{p(p+1)} \int u_{n}^{p+1} d x \\
& \leq \sup _{n} E\left(u_{n}\right)+q+\frac{1}{p(p+1)}\left|u_{n}\right|_{p+1}^{p+1} \\
& \leq A+q+A\|u\|^{\frac{p-1}{2}}|u|_{2}^{\frac{p+3}{2}} \leq A\left(1+\|u\|^{\frac{p-1}{2}}\right),
\end{aligned}
$$

where $A$ denotes various constants which are independent of $n$. Since $1<p<5$, the existence of the desired bound $B$ follows.

(iii) Let $\left\{u_{n}\right\}$ be a minimizing sequence for the problem $\left(I_{q}\right)$. Then we claim: there exists a constant $\eta>0$ such that $\left|u_{n}\right|_{p+1} \geq \eta$ for all sufficiently large $n$. We argue by contradiction: if no such $\eta>0$ exists, then $\liminf _{n \rightarrow \infty} \int\left|u_{n}\right|^{p+1} d x \leq 0$. Hence

$$
I_{q}=\lim _{n \rightarrow \infty} E\left(u_{n}\right) \geq-\liminf _{n \rightarrow \infty} \int\left|u_{n}\right|^{p+1} d x \geq 0,
$$

which contradicts (i). So, the claim is achieved.

Combining (ii) and Lemma 2.1, there exist a positive constant $\delta$ and a sequence $\left\{y_{n}\right\}$ of real numbers such that

$$
\int_{y_{n}-\frac{1}{2}}^{y_{n}+\frac{1}{2}}\left|u_{n}\right|^{p+1} d x \geq \delta
$$

for sufficiently large $n$. The proof of Lemma 3.1 is completed.

The next lemma will establish a subadditivity inequality which will be a crucial step in the proof of the existence minimizer for the problem $\left(I_{q}\right)$.

Lemma 3.2 For all $q_{1}, q_{2}>0, I_{q_{1}+q_{2}}<I_{q_{1}}+I_{q_{2}}$. 
Proof For given $u \in H^{1}(\mathbb{R}),|u|_{2}^{2}=q_{1}, \theta>0$, let $u_{\theta}(x)=\theta^{\frac{2}{p+1}} u\left(\theta^{\frac{p-1}{p+1}} x\right)$, where $\theta=\left(\frac{q_{2}}{q_{1}}\right)^{\frac{p+1}{5-p}}$. Then it follows that

$$
Q\left(u_{\theta}\right)=\frac{q_{2}}{q_{1}} Q(u)=q_{2},
$$

and

$$
E\left(u_{\theta}\right)=\left(\frac{q_{2}}{q_{1}}\right)^{\frac{p+3}{5-p}} E(u) .
$$

Hence we get

$$
I_{q_{2}}=\inf \left\{\left(\frac{q_{2}}{q_{1}}\right)^{\frac{p+3}{5-p}} E(u): Q(u)=q_{1}\right\}=\left(\frac{q_{2}}{q_{1}}\right)^{\frac{p+3}{5-p}} I_{q_{1}} .
$$

Now, from (3.2) and Lemma 3.1, we obtain for all $q_{1}, q_{2}>0$,

$$
I_{q_{1}+q_{2}}=\left(q_{2}+q_{1}\right)^{\frac{p+3}{5-p}} I_{1}<\left(q_{2}^{\frac{p+3}{5-p}}+q_{1}^{\frac{p+3}{5-p}}\right) I_{1}=I_{q_{1}}+I_{q_{2}}
$$

Now we formulate the following two theorems, which are special cases corresponding to Theorem 1.1 and Theorem 1.2, and give their proof with the aim of Lemma 3.1 and Lemma 3.2.

Theorem 3.1 Let $\alpha=1$ and $f(u)=\frac{1}{p} u^{p}$, where $1<p<5$. For any $q>0$, the set $G_{q}$ is not empty. Moreover, if $\left\{u_{n}\right\}$ is a minimizing sequence for the problem $\left(I_{q}\right)$, then there exist a sequence $\left\{y_{n}\right\} \subset \mathbb{R}$ and $g \in G_{q}$ such that $\left\{u_{n}\left(\cdot+y_{n}\right)\right\}$ contains a subsequence converging strongly in $H^{1}(\mathbb{R})$ to $g$, and

$$
\lim _{n \rightarrow+\infty} \inf _{g \in G_{q}}\left\|u_{n}-g\right\|=0 .
$$

Theorem 3.2 Let $\alpha=1$ and $f(u)=\frac{1}{p} u^{p}$, where $1<p<5$. For any $q>0$, the set $G_{q}$ is $H^{1}(\mathbb{R})$ stable with respect to equation (1.1), i.e., for any $\varepsilon>0$, there exists $\delta>0$ such that if

$$
\inf _{g \in G_{q}}\left\|u_{0}-g\right\|<\delta
$$

then the solution $u(x, t)$ to equation (1.1) with initial data $u_{0}$ satisfies

$$
\inf _{g \in G_{q}}\|u(t, \cdot)-g\|<\varepsilon
$$

for any $t \in[0, T)$.

Proof of Theorem 3.1 From (3.2), it is easy to check that $I_{q}$ is continuous on $(0, \infty)$. Let $\left\{u_{n}\right\}$ be a minimizing sequence for the problem $\left(I_{q}\right)$. By Lemma 3.1, there exist a positive constant $\delta$ and a sequence $\left\{y_{n}\right\}$ of real numbers such that

$$
\int_{y_{n}-\frac{1}{2}}^{y_{n}+\frac{1}{2}}\left|u_{n}\right|^{p+1} d x \geq \delta
$$

for sufficiently large $n$. 
Let us define $v_{n}=u_{n}\left(x+y_{n}\right)$. Hence $Q\left(V_{n}\right)=Q\left(u_{n}\right)=q, E\left(v_{n}\right)=E\left(u_{n}\right) \rightarrow I_{q}$, as $n \rightarrow \infty$, and

$$
\int_{-\frac{1}{2}}^{\frac{1}{2}}\left|v_{n}\right|^{p+1} d x=\int_{y_{n}-\frac{1}{2}}^{y_{n}+\frac{1}{2}}\left|u_{n}\right|^{p+1} d x \geq \delta>0 .
$$

Since $\left\{v_{n}\right\}$ is bounded in $H^{1}(\mathbb{R})$, by Lemma 3.1 , we may assume going, if necessary, to a subsequence

$$
\begin{array}{ll}
v_{n} \rightarrow g & \text { in } H^{1}(\mathbb{R}), \\
v_{n} \rightarrow g & \text { in } L_{\text {loc }}^{p+1}(\mathbb{R}), \\
v_{n} \rightarrow g & \text { a.e. on } \mathbb{R} .
\end{array}
$$

Hence, by (3.3), we get $g \neq 0$. And applying the Brezis-Lieb lemma [10], we have

$$
\begin{aligned}
& \left|v_{n}\right|_{2}^{2}=\left|v_{n}-g\right|_{2}^{2}+|g|_{2}^{2}, \\
& \left|v_{n}\right|_{p+1}^{p+1}=\left|v_{n}-g\right|_{p+1}^{p+1}+|g|_{p+1}^{p+1} .
\end{aligned}
$$

Now we show that $Q(g)=\frac{1}{2} \int g^{2} d x=q$. In the contrary case, $0<Q(g)=\lambda<q$. By (3.5), we obtain $\lim _{n \rightarrow \infty} q_{n}=\lim _{n \rightarrow \infty} Q\left(v_{n}-g\right)=q-\lambda$. Then it follows from (3.5) and (3.6) that

$$
\begin{aligned}
I_{q} & =E\left(v_{n}\right)+o(1) \geq \frac{1}{2}\left\|v_{n}\right\|^{2}-\frac{1}{2}\left|v_{n}\right|_{2}^{2}-\frac{1}{p(p+1)}\left|v_{n}\right|_{p+1}^{p+1}+o(1) \\
& =E\left(v_{n}-g\right)+E(g)+o(1) \geq I_{q_{n}}+I_{\lambda}+o(1) .
\end{aligned}
$$

Since $I_{q}$ is continuous on $(0, \infty)$, letting $n \rightarrow \infty$, we get $I_{q} \geq I_{q-\lambda}+I_{\lambda}$, which contradicts Lemma 3.2. Therefore $Q(g)=q$. It then follows from (3.5) that

$$
v_{n} \rightarrow g \quad \text { in } L^{2}(\mathbb{R})
$$

Applying the interpolation inequality, (3.4) and (3.8), we get

$$
v_{n} \rightarrow g \quad \text { in } L^{p+1}(\mathbb{R}) .
$$

Using the weak low semi-continuity of the norm in $H^{1}(\mathbb{R})$, we know that

$$
\begin{aligned}
I_{q} & \geq \frac{1}{2}\left\|v_{n}\right\|^{2}-\frac{1}{2}\left|v_{n}\right|_{2}^{2}-\frac{1}{p(p+1)}\left|v_{n}\right|_{p+1}^{p+1}+o(1) \\
& \geq \frac{1}{2}\|g\|^{2}-\frac{1}{2}\left|v_{n}-g\right|_{2}^{2}-\frac{1}{2}|g|_{2}^{2}-\frac{1}{p(p+1)}\left|v_{n}-g\right|_{p+1}^{p+1}-\frac{1}{p(p+1)}|g|_{p+1}^{p+1}+o(1) \\
& =E(g)-\frac{1}{2}\left|v_{n}-g\right|_{2}^{2}-\frac{1}{p(p+1)}\left|v_{n}-g\right|_{p+1}^{p+1}+o(1) .
\end{aligned}
$$

Letting $n \rightarrow \infty$, by (3.8) and (3.9), we obtain $E(g) \leq I_{q}$. On the other hand, it follows from $Q(g)=q$ that $E(g) \geq I_{q}$. Therefore $E(g)=I_{q}$, which implies that $g$ is a minimizer of the 
problem $\left(I_{q}\right)$ (i.e., $\left.G_{q} \neq \emptyset\right)$. Then it follows from (3.7), (3.8) and (3.9) that

$$
v_{n}=u_{n}\left(\cdot+y_{n}\right) \rightarrow g \quad \text { in } H^{1}(\mathbb{R}) .
$$

We prove $\lim _{n \rightarrow+\infty} \inf _{g \in G_{q}}\left\|u_{n}-g\right\|=0$ with an argument by contradiction. Assume that there exist $\varepsilon_{0}>0$ and a subsequence $\left\{u_{n_{k}}\right\}$ of $\left\{u_{n}\right\}$ such that

$$
\inf _{g \in G_{q}}\left\|u_{n_{k}}-g\right\| \geq \varepsilon_{0}>0
$$

for all $n_{k}$. With the result of the above proof, we obtain that there exist a subsequence of $\left\{u_{n_{k}}\right\}$, denoted again by $\left\{u_{n_{k}}\right\},\left\{y_{n_{k}}\right\} \subset \mathbb{R}$ and $g \in G_{q}$ such that

$$
u_{n_{k}}\left(\cdot+y_{n_{k}}\right) \rightarrow g \quad \text { in } H^{1}(\mathbb{R})
$$

Since $g\left(\cdot-y_{n_{k}}\right) \in G_{q}$,

$$
\left\|u_{n_{k}}-g\left(\cdot-y_{n_{k}}\right)\right\|=\left\|u_{n_{k}}\left(\cdot+y_{n_{k}}\right)-g\right\| \rightarrow 0 \quad \text { as } n_{k} \rightarrow+\infty,
$$

which contradicts (3.10).

An immediate consequence of Theorem 3.1 is that $G_{q}$ forms a stable set for the initialvalue problem for equation (1.1).

Proof of Theorem 3.2 We prove Theorem 3.2 with an argument by contradiction. Assume that the set $G_{q}$ is not $H^{1}(\mathbb{R})$-stable. Then there exist $\varepsilon_{0}>0,\left\{\psi_{n}\right\} \subset H^{1}(\mathbb{R})$ and a sequence of times $\left\{t_{n}\right\}$ such that

$$
\inf _{g \in G_{q}}\left\|\psi_{n}-g\right\|<\frac{1}{n}
$$

and

$$
\inf _{g \in G_{q}}\left\|u_{n}\left(\cdot, t_{n}\right)-g\right\| \geq \varepsilon_{0}
$$

for all $n$, where $u_{n}(x, t)$ solves equation (1.1) with $u_{n}(x, 0)=\psi_{n}$.

Equation (3.11) implies that

$$
E\left(\psi_{n}\right) \rightarrow I_{q}, \quad Q\left(\psi_{n}\right) \rightarrow q
$$

Choose $\left\{\mu_{n}\right\} \subset \mathbb{R}$ such that $Q\left(\mu_{n} \psi_{n}\right)=q$ for all $n$. Thus $\mu_{n} \rightarrow 1$ as $n \rightarrow+\infty$. Hence the sequence $v_{n}=\mu_{n} u_{n}\left(\cdot, t_{n}\right)$ satisfies $Q\left(v_{n}\right)=q$ and

$$
\lim _{n \rightarrow \infty} E\left(v_{n}\right)=\lim _{n \rightarrow \infty} E\left(u_{n}\left(\cdot, t_{n}\right)\right)=\lim _{n \rightarrow \infty} E\left(\psi_{n}\right)=I_{q} .
$$

Therefore $\left\{v_{n}\right\}$ is a minimizing sequence for the problem $\left(I_{q}\right)$. By Theorem 1.1, there exists $\left\{g_{n_{k}}\right\} \subset G_{q}$ such that

$$
\left\|v_{n_{k}}-g_{n_{k}}\right\|<\frac{\varepsilon_{0}}{2}
$$


for sufficiently large $n_{k}$. Since $\mu_{n} \rightarrow 1$ and $\left\|u_{n}\left(\cdot, t_{n}\right)\right\|$ is bounded, we derive from (3.12) and

$$
\begin{aligned}
\varepsilon_{0} & \leq\left\|u_{n_{k}}\left(\cdot, t_{n_{k}}\right)-g_{n_{k}}\right\| \\
& \leq\left\|u_{n_{k}}\left(\cdot, t_{n_{k}}\right)-\mu_{n_{k}} u_{n_{k}}\left(\cdot, t_{n_{k}}\right)\right\|+\left\|\mu_{n_{k}} u_{n_{k}}\left(\cdot, t_{n_{k}}\right)-g_{n_{k}}\right\| \\
& \leq\left(\left|\mu_{n_{k}}-1\right|\right)\left\|u_{n_{k}}\left(\cdot, t_{n_{k}}\right)\right\|+\frac{\varepsilon_{0}}{2} \leq \frac{3}{4} \varepsilon_{0}
\end{aligned}
$$

for sufficiently large $n_{k}$. (3.14) is a contradiction. Therefore, the set $G_{q}$ is $H^{1}(\mathbb{R})$-stable with respect to equation (1.1).

\section{The case for more general nonlinearities}

In this section, we consider (1.1) with $\alpha=1$ and more general nonlinearities $f$ satisfying condition (A). At first, we study the properties of the functional $I_{q}:(0, \infty) \rightarrow \mathbb{R}$ and the minimizing sequence of the problem $\left(I_{q}\right)$.

\section{Lemma 4.1}

(i) For any $q>0, I_{q}$ is finite and continuous on $(0, \infty)$. Moreover, each minimizing sequence for $\left(I_{q}\right)$ is bounded;

(ii) $I_{q} \leq 0$ for any $q>0$.

Proof (i) According to assumption (A), we observe that for each $\varepsilon>0$ there exists $C_{\varepsilon}>0$ such that

$$
|F(u)| \leq \varepsilon|u|^{2}+\varepsilon|u|^{\gamma+1}+C_{\varepsilon}|u|^{\alpha},
$$

where $2<\alpha<\gamma+1$. By the Sobolev embedding theorems and interpolation inequalities, we obtain

$$
\int_{-\infty}^{+\infty}|u|^{\alpha} d x \leq A\|u\|^{\frac{\alpha-2}{2}}|u|_{2}^{\frac{\alpha+2}{2}}
$$

and

$$
\int_{-\infty}^{+\infty}|u|^{\gamma+1} d x \leq A\|u\|^{\frac{\gamma-1}{2}}|u|_{2}^{\frac{\gamma+3}{2}}
$$

where $A>0$ is independent of $u$. Then using the Young inequality, we can derive from (4.2) and (4.3) that for all $\eta>0$, there exists $C_{\eta}>0$ such that

$$
\int_{-\infty}^{+\infty}|u|^{\alpha} d x \leq \eta\|u\|^{2}+C_{\eta}|u|_{2}^{\frac{2(\alpha+2)}{6-\alpha}}
$$

and

$$
\int_{-\infty}^{+\infty}|u|^{\gamma+1} d x \leq \eta\|u\|^{2}+C_{\eta}|u|_{2}^{\frac{2 \gamma+6}{5-\gamma}}
$$


Let $u \in H^{1}(\mathbb{R})$ such that $Q(u)=q$. It follows from (4.1), (4.4) and (4.5) that

$$
\begin{aligned}
E(u) & =\frac{1}{2}\|u\|^{2}-\frac{1}{2} \int_{-\infty}^{+\infty} u^{2} d x-\int_{-\infty}^{+\infty} F(u) d x \\
& \geq \frac{1}{2}\|u\|^{2}-\frac{1}{2}|u|_{2}^{2}-\varepsilon|u|_{2}^{2}-\varepsilon \eta\|u\|^{2}-\varepsilon C_{\eta}|u|_{2}^{\frac{2 \gamma+6}{5-\gamma}}-C_{\varepsilon} \eta\|u\|^{2}-C_{\varepsilon} C_{\eta}|u|_{2}^{\frac{2(\alpha+2)}{6-\alpha}} \\
& \geq\left(\frac{1}{2}-\varepsilon \eta-C_{\varepsilon} \eta\right)\|u\|^{2}-C_{\varepsilon, \eta, q},
\end{aligned}
$$

where $C_{\varepsilon, \eta, q}$ is a positive constant dependent only on $\varepsilon$ and $\eta$ for given $q>0$. Choosing $\varepsilon>0$ and $\eta>0$ such that $\frac{1}{2}-\varepsilon \eta-C_{\varepsilon} \eta>0$, we see that $I_{q}>-\infty$.

Since $|\theta u|_{2}^{2}=\theta^{2}|u|_{2}^{2}$ for $\theta>0$, it is easy to check that $I_{q}$ is continuous on $(0, \infty)$.

Let $\left\{u_{n}\right\}$ be a minimizing sequence for the problem $\left(I_{q}\right)$. From (4.6) and the fact that $I_{q}$ is finite, we know that $\left\{u_{n}\right\}$ is bounded in $H^{1}(\mathbb{R})$.

(ii) For given $u \in H^{1}(\mathbb{R})$ such that $Q(u)=q$, let $u_{\theta}(x)=\frac{1}{\sqrt{\theta}} u\left(\frac{x}{\theta}\right)$ for $\theta>0$. We obtain that

$$
\left|u_{\theta}\right|_{2}^{2}=|u|_{2}^{2}=q
$$

and

$$
E\left(u_{\theta}\right)=\frac{1}{2 \theta^{2}} \int_{-\infty}^{+\infty}\left(u_{x}\right)^{2} d x-\theta \int_{-\infty}^{+\infty} F\left(\frac{1}{\sqrt{\theta}} u\right) d x
$$

Combining (4.1), (4.7) and (4.8), we obtain

$$
I_{q} \leq E\left(u_{\theta}\right) \rightarrow 0 \quad \text { as } \theta \rightarrow+\infty
$$

Lemma 4.2 Suppose that $I_{q_{0}}<0$ for some $q_{0}>0$. Then the following two properties hold:

(i) $\frac{I_{q}}{q}$ is non-increasing on $(0,+\infty)$ and $\lim _{q \rightarrow 0^{+}} \frac{I_{q}}{q}=0$;

(ii) there exists $q_{1} \leq q_{0}$ such that

$$
\frac{I_{q}}{q}>\frac{I_{q_{0}}}{q_{0}} \quad \text { for } q \in\left(0, q_{1}\right)
$$

Proof First we observe that if $\sigma>0$ and $\beta>0$ with $Q(u)=\beta$ and $u_{\sigma}(x)=u\left(\frac{1}{\sigma} x\right)$, then $Q\left(u_{\sigma}\right)=\sigma \beta$ and

$$
E\left(u_{\sigma}\right)=\frac{1}{2 \sigma} \int_{-\infty}^{+\infty}\left(u_{x}\right)^{2} d x-\sigma \int_{-\infty}^{+\infty} F(u) d x .
$$

Consequently, for $q_{1}>0$ and $q_{2}>0$, we have

$$
I_{q_{2}}=\inf \left\{\frac{q_{1}}{2 q_{2}} \int_{-\infty}^{+\infty}\left(u_{x}\right)^{2} d x-\frac{q_{2}}{q_{1}} \int_{-\infty}^{+\infty} F(u) d x, Q(u)=q_{1}\right\} .
$$

If $q_{1}>q_{2}>0$, then for each $\varepsilon>0$, there exists $u \in H^{1}(\mathbb{R})$ with $Q(u)=q_{1}$ such that

$$
I_{q_{2}}+\varepsilon>\frac{1}{2} \frac{q_{1}}{q_{2}} \int_{-\infty}^{+\infty}\left(u_{x}\right)^{2} d x-\frac{q_{2}}{q_{1}} \int_{-\infty}^{+\infty} F(u) d x>\frac{q_{2}}{q_{1}} E(u) \geq \frac{q_{2}}{q_{1}} I_{q_{1}} .
$$

This inequality yields $\frac{I_{q_{2}}}{q_{2}} \geq \frac{I_{q_{1}}}{q_{1}}$ for $0<q_{2}<q_{1}$. 
Since $I_{q} \leq 0$ for all $q>0$, we see that

$$
\lim _{q \rightarrow 0^{+}} \frac{I_{q}}{q}=A \leq 0
$$

We claim that $A=0$. Letting $\varepsilon=q^{2}, 0<q \leq q_{0}$, from (4.9), there exists $u^{(q)} \in H^{1}(\mathbb{R})$ with $Q\left(u^{(q)}\right)=q_{0}$ such that

$$
\begin{aligned}
I_{q}+q^{2} & \geq \frac{1}{2} \frac{q_{0}}{q} \int_{-\infty}^{+\infty}\left(u_{x}^{(q)}\right)^{2} d x-\frac{q}{q_{0}} \int_{-\infty}^{+\infty} F\left(u^{(q)}\right) d x \\
& \geq \frac{q}{q_{0}}\left[\frac{1}{2} \int_{-\infty}^{+\infty}\left(u_{x}^{(q)}\right)^{2} d x-\int_{-\infty}^{+\infty} F\left(u^{(q)}\right) d x\right] .
\end{aligned}
$$

It follows from (4.6) and (4.10) that

$$
I_{q}+q^{2} \geq \frac{q}{q_{0}}\left(C_{1}\left(q_{0}\right) \int_{-\infty}^{+\infty}\left(u_{x}^{(q)}\right)^{2} d x-C_{2}\left(q_{0}\right)\right)
$$

where $C_{1}\left(q_{0}\right)>0$ and $C_{2}\left(q_{0}\right)>0$ are constants independent of $q_{0}$. Hence we obtain $q_{0}^{2} \geq$ $C_{1}\left(q_{0}\right) \int_{-\infty}^{+\infty}\left(u^{(q)}\right)_{x}^{2} d x-C_{2}\left(q_{0}\right)$, which implies

$$
\int_{-\infty}^{+\infty}\left(u_{x}^{(q)}\right)^{2} d x \leq C_{3}\left(q_{0}\right)
$$

where $C_{3}\left(q_{0}\right)$ is dependent only on $q_{0}$. Combining (4.1), (4.4), (4.5) and (4.11), we also get

$$
\left|\int_{-\infty}^{+\infty} F\left(u^{(q)}\right) d x\right| \leq C_{4}\left(q_{0}\right)
$$

where $C_{4}\left(q_{0}\right)$ is dependent only on $q_{0}$.

We claim that

$$
\int_{-\infty}^{+\infty}\left(u_{x}^{(q)}\right)^{2} d x \rightarrow 0 \quad \text { as } q \rightarrow 0^{+}
$$

Indeed, if there exist $\varepsilon_{0}>0$ and $q_{n} \rightarrow 0^{+}$such that $\int_{-\infty}^{+\infty}\left(u_{x}^{\left(q_{n}\right)}\right)^{2} d x \geq \varepsilon_{0}$, then by (4.11) and (4.12), we obtain

$$
\frac{I_{q_{n}}}{q_{n}}+q_{n} \geq \frac{q_{0} \varepsilon_{0}}{2} \frac{1}{q_{n}^{2}}-\frac{1}{q_{0}} C_{4}\left(q_{0}\right) \rightarrow+\infty \quad \text { as } q_{n} \rightarrow 0^{+}
$$

which contradicts $\lim _{q \rightarrow 0^{+}} \frac{I_{q}}{q}=A \leq 0$. Therefore (4.13) is achieved and this implies that $\lim _{q \rightarrow 0^{+}} \int_{-\infty}^{+\infty} F\left(u^{(q)}\right) d x=0$ and, consequently,

$$
\frac{I_{q}}{q}+q \geq-\frac{1}{q_{0}} \int_{-\infty}^{+\infty} F\left(u^{(q)}\right) d x \rightarrow 0 \quad \text { as } q \rightarrow 0^{+}
$$

This shows that $\lim _{q \rightarrow 0^{+}} \frac{I_{q}}{q}=0$.

(2) We observe that $\lim _{q \rightarrow 0^{+}} \frac{I_{q}}{q}=0>\frac{I_{q_{0}}}{q_{0}}$, which implies (ii). 
Then we establish a subadditivity inequality similar to Lemma 3.2 with the aim of Lemma 4.2.

Lemma 4.3 Suppose that $I_{q_{0}}<0$ for some $q_{0}>0$. Then there exists $0<q^{*} \leq q_{0}$ such that $I_{q^{*}}<I_{q^{*}-q}+I_{q}$ for $0<q<q^{*}$.

Proof According to Lemma 4.2, the set

$$
\left\{q_{1} \mid q_{1} \leq q_{0} \text { and } \frac{I_{q}}{q}>\frac{I_{q_{0}}}{q_{0}} \text { or each } q \in\left(0, q_{1}\right)\right\}
$$

is nonempty. We define

$$
q^{*}=\sup \left\{q_{1} \mid q_{1} \leq q_{0} \text { and } \frac{I_{q}}{q}>\frac{I_{q_{0}}}{q_{0}} \text { for each } q \in\left(0, q_{1}\right)\right\} .
$$

It follows from the continuity of $I_{q}$ and $\lim _{q \rightarrow 0^{+}} \frac{I_{q}}{q}=0$ that $0<q^{*} \leq q_{0}$,

$$
\begin{aligned}
& I_{q^{*}}=\frac{q^{*}}{q_{0}} I_{q_{0}}<0, \\
& I_{q}>\frac{q}{q_{0}} I_{q_{0}} \text { for all } q \in\left(0, q^{*}\right) .
\end{aligned}
$$

Therefore,

$$
I_{q^{*}}=\frac{q^{*}}{q_{0}} I_{q_{0}}=\frac{q^{*}-q}{q_{0}} I_{q_{0}}+\frac{q}{q_{0}} I_{q_{0}}<I_{q^{*}-q}+I_{q},
$$

for all $q \in\left(0, q^{*}\right)$.

Now we give the proof of Theorem 1.1 with the aim of Lemma 4.1, Lemma 4.2 and Lemma 4.3. Since the proof of Theorem 1.1 is similar to that of Theorem 3.1, we only give the sketch of the proof.

Proof of Theorem 1.1 Let $\left\{u_{n}\right\}$ be a minimizing sequence of $I_{q^{*}}$, where $q^{*}$ is defined in (4.14). Since $\left\{u_{n}\right\}$ is bounded, we may assume

$$
\begin{array}{ll}
u_{n} \rightarrow u & \text { in } H^{1}(\mathbb{R}), \\
u_{n} \rightarrow u & \text { a.e. on } \mathbb{R} .
\end{array}
$$

First, we consider the case $u=0$. In this case, by Lemma 2.2, either

(a) $u_{n} \rightarrow 0$ in $L^{s}(\mathbb{R})$ for $2<s<\infty$, or

(b) there exists a sequence $\left\{y_{n}\right\} \subset \mathbb{R}$ such that

$$
v_{n}(x)=u_{n}\left(x+y_{n}\right) \rightarrow g \neq 0 \quad \text { in } H^{1}(\mathbb{R}) .
$$

In the case (a), combining Lemma 2.2 and condition (A), we obtain

$$
\lim _{n \rightarrow \infty} \int_{-\infty}^{+\infty} F\left(u_{n}\right) d x=0
$$


and, consequently,

$$
I_{q^{*}}=\lim _{n \rightarrow \infty} E\left(u_{n}\right)=\lim _{n \rightarrow \infty}\left[\frac{1}{2} \int_{-\infty}^{+\infty}\left(u_{n x}\right)^{2} d x-\int_{-\infty}^{+\infty} F\left(u_{n}\right) d x\right] \geq 0
$$

which contradicts Lemma 4.1. Hence (b) holds. Then it follows from Lemma 2.3 and Lemma 4.3 that $g$ is the minimizer for the problem $\left(I_{q^{*}}\right)\left(\right.$ i.e., $\left.G_{q^{*}} \neq \emptyset\right)$ and the result of Theorem 1.1 holds. The proof is similar to that of Theorem 3.1, we omit the details.

If $u \neq 0$, we repeat the previous argument in the case (b) to obtain the result of Theorem 1.1.

Proof of Theorem 1.2 Theorem 1.2 is an immediate result of Theorem 1.1. We can prove it with an argument similar to that of Theorem 3.2. Here, we omit the details of the proof.

\section{The case for $0<\alpha<1$}

In this section, we only consider the case of $0<\alpha<1$, i.e., we consider the existence and stability of solitary waves for the fractional Korteweg-de Vries equations with general nonlinearities. At first, we give the definition of $H^{\alpha}(\mathbb{R})$. The fractional order Sobolev space $H^{\alpha}(\mathbb{R})$ is defined by

$$
H^{\alpha}(\mathbb{R}):=\left\{u ; u: \mathbb{R} \rightarrow \mathbb{C}, u \in L^{2}(\mathbb{R}) \text { and } \mathcal{F}^{-1}\left[\left(1+|\xi|^{2}\right)^{\frac{\alpha}{2}} \mathcal{F} u\right] \in L^{2}(\mathbb{R})\right\}
$$

whose norm is given by

$$
\|\cdot\|_{\alpha, 2}=\left|\mathcal{F}^{-1}\left[\left(1+|\xi|^{2}\right)^{\frac{\alpha}{2}} \mathcal{F} \cdot\right]\right|_{2}
$$

Since the functionals

$$
E(u)=\int_{-\infty}^{+\infty}\left[\frac{1}{2}\left|(-\triangle)^{\frac{\alpha}{2}} u\right|^{2}-F(u)\right] d x
$$

and

$$
Q(u)=\frac{1}{2} \int_{-\infty}^{+\infty} u^{2} d x
$$

are two conserved quantities with (1.1), for studying the existence of solitary wave solutions to (1.1), by the variational methods, the solitary wave solutions to the equation (1.1) will be founded as minimizers of

$$
I_{q}:=\inf \left\{E(u) ; u \in H^{1}(\mathbb{R}), Q(u)=q\right\},
$$

where $q>0$. Denote the set of minimizers of the problem $\left(I_{q}\right)$ by

$$
G_{q}:=\left\{u ; u \in H^{1}(\mathbb{R}), E(u)=I_{q}, Q(u)=q\right\} .
$$

Similar to Lemma 4.1 and Lemma 4.3, we obtain the following two lemmas. 


\section{Lemma 5.1}

(i) $I_{q}^{\infty}$ and $I_{q}$ are finite and continuous on $(0,+\infty)$; moreover, for any $q>0$, each minimizing sequence for the problem $\left(I_{q}^{\infty}\right)$ or $\left(I_{q}\right)$ is bounded in $H^{\alpha}(\mathbb{R})$;

(ii) $I_{q}^{\infty} \leq 0$ for any $q>0$.

Lemma 5.2 If $I_{q_{0}}^{\infty}<0$ for some $q_{0}>0$, then there exists $q_{1}, 0<q_{1} \leq q_{0}$, such that

$$
I_{q_{1}}^{\infty}<I_{q_{1}-q}^{\infty}+I_{q}^{\infty} \text { for } 0<q<q_{1} .
$$

Applying the above two lemmas and commutator estimates [5, Lemma 2.5], we prove Theorem 1.3 and Theorem 1.4 by similar steps to those given in Section 4 . Here we omit the details of Theorem 1.3 and Theorem 1.4.

\section{Competing interests}

The author declares that they have no competing interests.

\section{Acknowledgements}

The work was supported in part by Special Fund of Fundamental Scientific Research Business Expense for Higher Schoo of Central Government (projects for young teachers, No. ZY20110226) and the National Natural Science Foundation of China (No. 41276020)

Received: 31 March 2013 Accepted: 16 April 2013 Published: 10 May 2013

\section{References}

1. Korteweg, DJ, de Vries, G: On the change of form of long waves advancing in a rectangular canal, and on a new type of long stationary waves. Philos. Mag. 39, 422-443 (1895)

2. Benjamin, TB: The stability of solitary waves. Proc. R. Soc. Lond. Ser. A 328, 153-183 (1972)

3. Pelinovsky, E: Analytical models of tsunami generation by submarine landslides. In: Submarine Landslides and Tsunamis. Nato Science Series, vol. 21, pp. 111-128. Kluwer Academic, Dordrecht (2003)

4. Albert, JP: Concentration compactness and the stability of solitary-wave solutions to nonlocal equations. In: Applied Analysis (Baton Rouge, LA, 1996) Contemporary Mathematics, vol. 221, pp. 1-29. Am. Math. Soc., Providence (1999)

5. Guo, B, Huang, D: Existence and stability of standing waves for nonlinear fractional Schrödinger equations. J. Math. Phys. 53, $083702(2005)$

6. Cazenave, T, Lions, PL: Orbital stability of standing waves for some nonlinear Schrödinger equations. Commun. Math. Phys. 85, 549-561 (1982)

7. Lions, PL: The concentration-compactness principle in the calculus of variation. The locally compact case. I. Ann. Inst. Henri Poincaré, Anal. Non Linéaire 1, 109-145 (1984)

8. Lions, PL: The concentration-compactness principle in the calculus of variation. The locally compact case. II. Ann. Inst. Henri Poincaré, Anal. Non Linéaire 1, 223-283 (1984)

9. Adams, RA: Sobolev Space. Academic Press, New York (1975)

10. Willem, M: Minimax Theorems. Birkhäuser, Boston (1996)

doi:10.1186/1687-2770-2013-121

Cite this article as: Hong: Existence and stability of solitary waves for the generalized Korteweg-de Vries equations. Boundary Value Problems 2013 2013:121.

\section{Submit your manuscript to a SpringerOpen ${ }^{\ominus}$ journal and benefit from:}

- Convenient online submission

- Rigorous peer review

- Immediate publication on acceptance

Open access: articles freely available online

- High visibility within the field

- Retaining the copyright to your article 